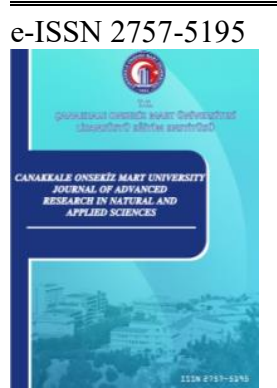

Çanakkale Onsekiz Mart University

Journal of Advanced Research in Natural and Applied Sciences

Open Access

\title{
Boundary Extraction Based on Dual Stream Deep Learning Model in High Resolution Remote Sensing Images
}

\author{
Özgün Akçay ${ }^{1 *}$, Ahmet Cumhur Kınacr ${ }^{2}$, Emin Özgür Avşar ${ }^{1}$, Umut Aydar $^{1}$ \\ ${ }^{1}$ Department of Geomatics Engineering, Faculty of Engineering, Çanakkale Onsekiz Mart University, Çanakkale, Turkey \\ ${ }^{2}$ Department of Computer Engineering, Faculty of Engineering, Çanakkale Onsekiz Mart University, Çanakkale, Turkey

$\begin{array}{lr}\text { Article History } \\ \text { Received: } & 07.04 .2021 \\ \text { Accepted: } & 23.06 .2021 \\ \text { Published: } & 20.09 .2021\end{array}$ \\ Research Article

\begin{abstract}
Boundary extraction in remote sensing has an important task in studies such as environmental observation, risk management and monitoring urban growth. Although significant progress has been made in the different calculation methods proposed, there are issues that need improvement, especially in terms of accuracy, efficiency and speed. In this study, dual stream network architecture of three different models that can obtain boundary extraction by using normalized Digital Surface Model (nDSM), Normalized Difference Vegetation Index (NDVI) and Near-Infrared (IR) band as the second stream, was explained. Model I is designed as the original HED, whereas the second stream of Model II, III, and IV use nDSM, nDSM + NDVI and nDSM + NDVI + IR, respectively. Thus, by comparing the models trained based on different data combinations, the contribution of different input data to the success of boundary extraction was revealed. For the training of the models, boundary maps produced from The International Society for Photogrammetry and Remote Sensing (ISPRS) Potsdam data set and input datasets augmented by rotation, mirroring and rotation were used. When the test results obtained from two-stream and multidata-based models are evaluated, $11 \%$ higher recall values have achieved with Model IV compared to the original HED. The outcomes clearly revealed the importance of using multispectral band, height data and vegetation information as input data in boundary extraction beside commonly used RGB images.
\end{abstract}

Keywords - Boundary extraction, deep learning, orthophotos, photogrammetry, remote sensing

\section{Introduction}

The studies for automatic extraction of natural and man-made object boundaries from optical images obtained from aerial cameras and satellite sensors have been carried out by Geomatics and Computer vision disciplines for many years and are still up-to-date with increasing interest. Automatic object extraction, which is particularly one of the main topics in Photogrammetry and Remote Sensing (RS) disciplines, has a very important task in environmental monitoring, risk management, precision agriculture, and effective database updating for Geographic Information Systems. Different methods have been used in the semantic classification studies that started in the 80's until today. Cheng et al. (2016) includes a comprehensive review of the studies conducted in this field. Although significant progress has been made with the different algorithms and methods, there are issues that need improvement, especially in terms of accuracy, efficiency and speed.

While the extraction methods continue to improve, on the other hand, the increasing image resolutions in parallel with the developments in sensor technologies have made new researches in semantic classification studies necessary. Since the early satellite images with low spatial resolution generally did not allow object

\footnotetext{
1 (D) akcay@comu.edu.tr

2 (D) cumhur.kinaci@comu.edu.tr

3 (1D) ozguravsar@comu.edu.tr

4 (D) umutaydar@ comu.edu.tr

${ }^{*}$ Corresponding Author
} 
extraction, semantic classification studies were mostly carried out to obtain regional features. However, thanks to the increased spatial resolutions (SPOT, Pleiades, aerial photographs, etc.) as the result of the development in image technology, it became possible to automatically extract objects and determine object boundaries; studies have focused on these areas. On the other hand, the visibility of complex object geometries with high resolution necessitated the development of semantic classification methods. Automatic object extraction, which will form a basis for high-accuracy studies such as engineering projects, cadastral studies, through highresolution remote sensing images and aerial photographs, is one of the most challenging issues in this field (Han et al., 2017; Altınoluk et al., 2020).

In recent years, it has been observed that deep convolutional neural networks (DCNN) have an important place in semantic classification and object boundary extraction studies on remote sensing images. According to many researchers, the success of deep networks stems from their ability to learn end-to-end efficient matching between raw images and class labels (Kinzie \& Kuh, 2004; Marmanis et al., 2018). Many classifications and object extraction studies have been carried out on remote sensing images by using Deep Networks with different architectures. Guo et al. (2018) and Yuan et al. (2021) discusses the many deep learning methods used in remote sensing in detail. In these studies, the errors occurring especially at the object boundaries revealed the need for studies on the extraction of the boundaries that determine the linear object edges.

Hariharan et al. (2011) proposed a method using an image-based weighted feature vector with the object definition frame, also known as the activation window in order to determine the boundary lines belonging to the object. Then, the final boundary lines of the whole image were formed by combining the border lines obtained from the detected objects in different categories. The combination used in this study has formed the basis for deep learning methods in determining object boundaries. Shen et al. (2015) used a customized training method by classifying line data in convolutional networks into subclasses depending on their shapes. In this method, positive loss function based on all class assignments is used and the loss is dealt out among subclasses. Later, because the shapes of the sub-classes are different from each other, a different model parameter was determined for each subclass and these classes were trained.

The one of the most cited studies in the literature for border detection is the Holistically-nested Edge Detection (HED) method (Xie \& Tu, 2015). In HED, holistic expression means that a network can obtain edge map directly from image input, and nested expression represents using side outputs. HED, which was created using the Deep Supervised Network structure (Lee et al., 2015) and pre-trained VGG network parameters, has provided significant improvements in object boundaries and edge detection. Kokkinos (2015) developed HED with a multi-scale approach that designs different dimensions of the image pyramid as a single HED.

Edge extraction is considered with different task descriptions in networks that can perform multiple tasks instead of a single task. In their study, Kokkinos (2017) developed a network called UBERNet, which is suitable for the need for training data in different content for different tasks including edge extraction and the need for increased computing load due to multitasking. UBERNet is a fully convolutional network designed with task-oriented special layers placed on the basis of the VGG network. Network highlights can be expressed as follows: Bringing together the upper layers and middle layers by skipping layers, and making use of the spatial knowledge of lower level neurons in edge extraction, reducing slow learning rates with batch normalization in the middle layers and applying extended convolution. Chen et al. (2016) used the low-level layers of the DeepLab network (Chen et al., 2014) for edge detection with semantic segmentation as well as edge estimation, and obtained the improved semantic map from the results with an end-to-end architecture. On the other hand, Dai et al. (2016) performed a semantic segmentation that is sensitive to each object instance, in other words, identifying the object instances separately. This architecture, which was developed as multitasking and end-to-end, revealed the object examples of a class by sharing the property maps of three separate tasks. 
In another study on segment boundaries, Ultrametric Contour Map was obtained by combining the horizontal and vertical orientations of multi-scale border lines and feature maps created as side outputs of the convolution network (Maninis et al., 2017). Marmanis et al. (2018) tried to minimize the edge defects that occur as a result of semantic segmentation by defining the HED and SegNet architecture for the remote sensing images in an integrated network for spectral, DSM and nDSM datasets in a multi-scale. In this study, double-stream HED$\mathrm{H}$ and Segnet- $\mathrm{H}$ architectures are proposed instead of classical architectures by including height data as the second input data in education. CaseNet, which aims to determine the classes to which the borderlines belong, was developed on the layer jump and ResNet architecture, which shares the category-dependent edge activations in the top convolution layer and combines them with the lower layer property map (Yu et al., 2017). This study identified edge semantics despite some class inconsistencies. Bokhovkin \& Burnaev (2019) proposes a novel loss function, namely a differentiable surrogate of a metric accounting accuracy of boundary detection. In the study, validation of proposed loss function is carried out with various modifications of UNet architecture on a synthetic dataset as well as real-world data. Alam et. al. (2021) proposes an improved Encoder-Decoder deep learning model that combines SegNet with index pooling and adapted U-net for suitable for multi-targets semantic segmen-tation of RS images. In the study, it is stated that by index pooling it is possible to obtain a more clear details for the segmentation of the edges.

However, recent related works have not shown how remote sensing-oriented datasets such as near-infrared band, height information, vegetation index might contribute to boundary extraction in deep learning. In this study, a dual stream network architecture that is able to extract object boundaries utilizing nDSM, NDVI and IR as additional data types for the RGB band information is proposed. In addition, the contribution of data types to the success of boundary inference was revealed by comparing the models trained based on different data combinations.

\section{Data Preparation}

In this study, The Potsdam dataset, which has been accessed within the scope of the International Society for Photogrammetry and Remote Sensing - Working Group III/4 was used (Rottensteiner et al., 2012). The datasets include true orthophotos, Digital Surface Models (DSMs) and ground truths produced from true orthophoto mosaic. True orthophotos and DSMs are in $5 \mathrm{~cm}$ ground sampling distances, Universal Transverse Mercator (UTM) projection and WGS84 datum. True-orthophotos and DSMs are available in 38 pieces of $6000 \times 6000$ pixel sizes, and 24 training and 14 test ground truths are available in tiff image format. True orthophotos are presented as three different combinations of four bands with 8-bit spectral resolution: RedGreen-Blue-Infrared (RGBIR), Infrared-Red-Green (IRRG) and Red-Green-Blue (RGB). In addition to DSMs presented with values encoded in 32-bit float data type, Normalized Digital Surface Models (nDSMs) produced by subtracting surface heights from digital elevation models are also included as an image data in jpeg image format.

In the preparation of the data, considering GPU memory limits and unintentional memorization, the appropriate image size for the input data was determined as 800 x 800 pixels with a 150 pixels overlap. As a result of this approach, 81 input data were generated from each image of 6000 x 6000 pixels. On the other hand, data augmentation should be considered to expand limited datasets as it can improve the performance of deep learning models (Shorten and Khoshgoftaar, 2019). Particularly, the brightness values of RGB and IR data were changed by $\pm 50 \%$, by predicting the effect of different image acquisition angles and reflection differences. Figure 1 depicts the effect of rotation, mirroring and contrast changes for an RGB image after augmentation was implemented. RGB, nDSM, NDVI, IR and label data were mirrored and the original and mirrored images were rotated 90, 180 and 270 degrees. Therefore, the original RGB, IR datasets and nDSM, NDVI, label datasets were augmented 24 times and 8 times, respectively. The number of datasets resulting from this approach is given in Table 1 . All input data preparation processes were coded and carried out in GNU Octave environment. 
Four different datasets RGB, nDSM, NDVI and IR extracted from the Potsdam benchmark were defined as input sets in the study. Single band IR datasets were sliced from the RGBIR images and NDVI datasets were calculated and produced using the first and fourth bands of RGBIR true orthophotos. To produce labels for boundary data, the segmented label data containing border information have been processed.

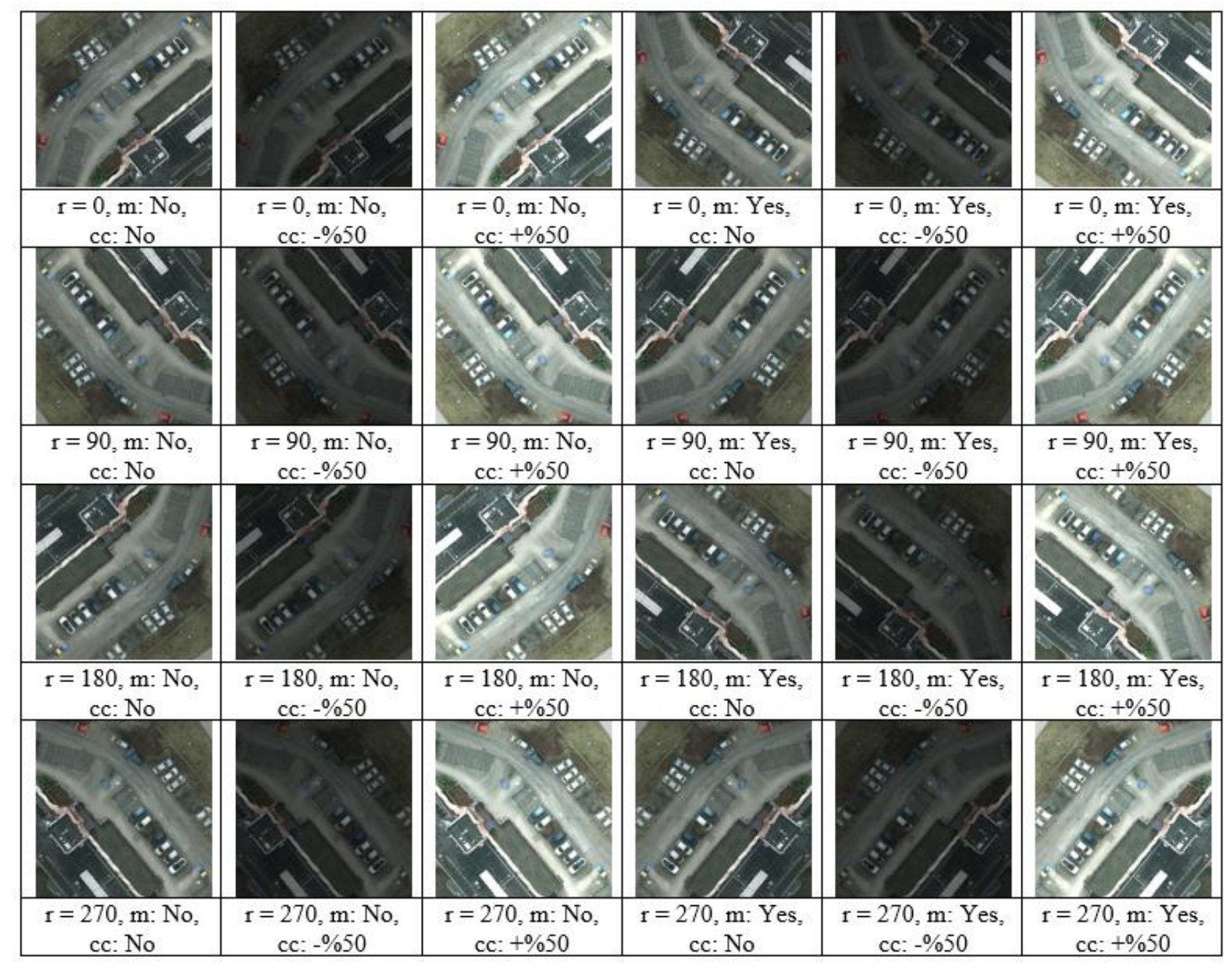

Figure 1. Augmentation result of an RGB image for rotation (r) in degrees, mirroring (m) and contrast change (cc)

Table 1

Number of augmented samples

\begin{tabular}{lllll}
\hline Data type & Rotation & Mirroring & Contrast & Number of samples \\
\hline RGB & + & + & + & 73872 \\
nDSM & + & + & - & 24624 \\
NDVI & + & + & - & 24624 \\
IR & + & + & + & 73872 \\
Label & + & + & - & 24624 \\
Total & & & & 221616 \\
\hline
\end{tabular}

\section{Methods}

Four models, Model I, II, III and IV have been discussed in this paper to determine data contribution to boundary extraction. Model I ( Model II, III and IV are designed with one parallel stream getting additional inputs beside Model 1. Some 
combinations of nDSM, NDVI and IR are utilized as inputs of Model II, III, and IV in their second streams, while RGB true orthophotos data is used as input in their first stream as shown in Table 2 . Marmanis et al. (2018) also proposed a dual stream HED that is summarized in Figure 2; however they fed their model with only DSM datasets at second branch.

Table 2

Input datasets for the models

\begin{tabular}{lll}
\hline Model no & First stream & Second stream \\
\hline I & RGB & - \\
II & RGB & nDSM \\
III & RGB & nDSM + NDVI \\
IV & RGB & nDSM + NDVI + IR \\
\hline
\end{tabular}

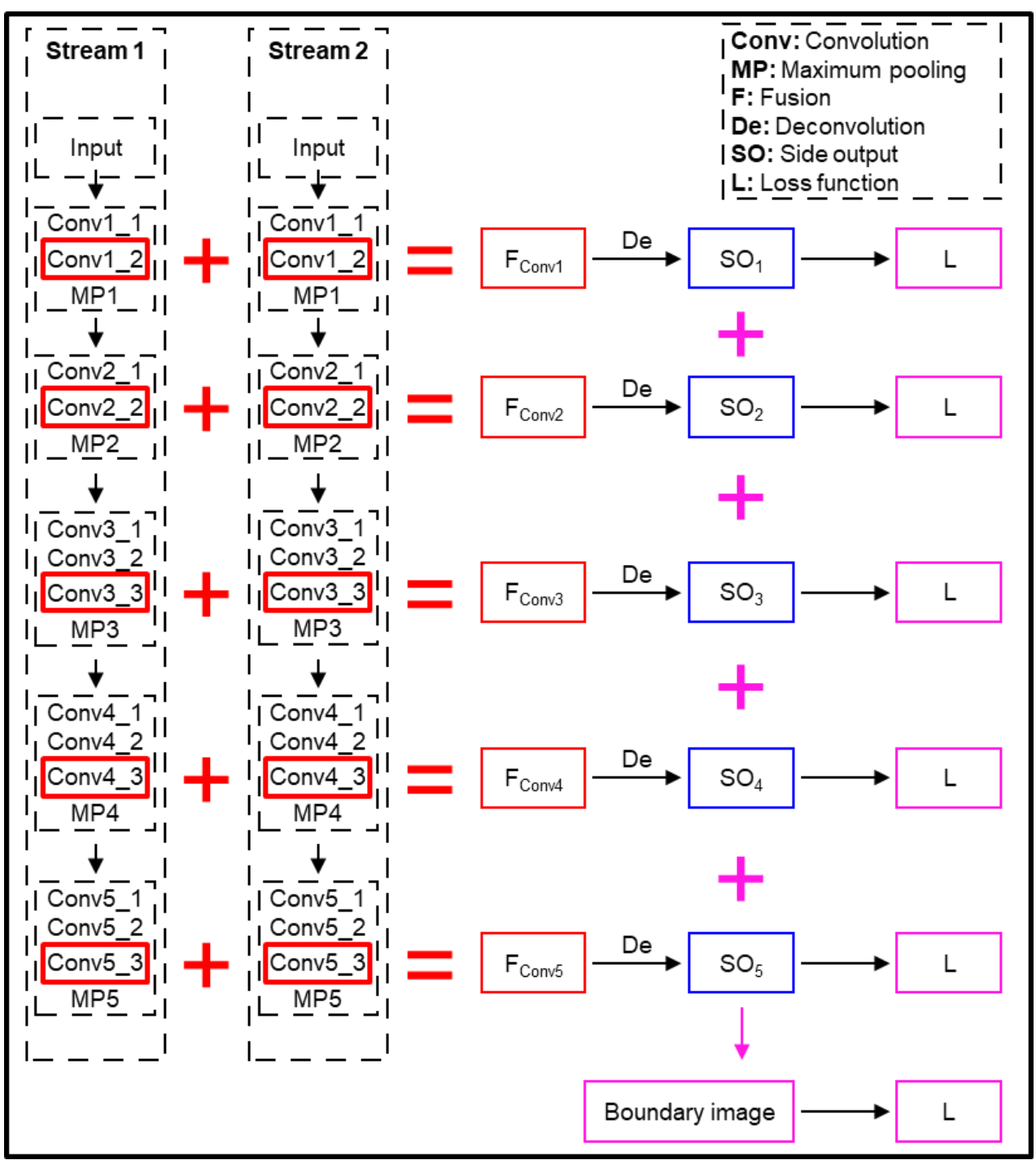

Figure 2. Dual stream model for object boundaries

By using different data types in the training of models, the effect of data other than RGB on the performance of the neural network was measured. The first stream was started with VGG-16 weights, but since there were no trained models of the input data to be used in the second stream of Models 2 and 3, the random initialization method was used initially. Since the input of the second flow of Model 4 is three-layered, the starting weights 
were transferred from VGG-16 in this flow as well. Dual Stream HED (DS-HED) treats the convolution layers before each maximum pooling layer in both streams as a side output layer. Then, deconvolution and balanced cross-entropy loss function are applied to the side output layers, respectively, and the object boundary map is produced by combining these outputs as mentioned in Xie and $\mathrm{Tu}(2015)$. Two-stream net architecture consists of 26 convolutions, 10 maximum pooling, and 5 deconvolutions. All tests were carried out on a desktop computer including Intel(R) Core(TM) i7-7820X CPU @ 3.60GHz, 2xGeForce RTX 2080 Ti 128 GB RAM.

\section{Results and Discussion}

Determining the most suitable model requires long processing times due to the very high size of the training data. In order to make a quick comparison between the performance of different models, an evaluation was made using training data sets produced from two selected layouts instead of the entire training data set. Initial model training experiments were accomplished by selecting the layouts 2-10 and 5-12 representing different characteristics such as buildings, roads and vegetation. Among the HED models trained for class boundaries inference, Model I trained with RGB was used as the base comparison model. The performances of Model II and Model III that were tested with nDSM and NDVI datasets added to the second stream, including RGB in the first stream, examine the success according to Model I. With Model IV, all data of nDSM, NDVI, IR were used for the second flow of HED. The graphs of the training and validation loss values generated during the training of all models depending on the increasing number of epochs are shown in Figure $\underline{3}$. Since the number of training data was kept low in the preliminary evaluation, some deviations were observed in validation values due to the number of epochs. Table $\underline{3}$, on the other hand, explains the number of data, the number of epochs and the accuracy results of the training based on the map data of all models 2-10 and 5-12.

True Positive (TP), True Negative (TN), False Positive (FP) and False Negative (FN) numerical values were considered to determine the error evaluation and accuracy rates between the predicted results and the actual values. With these numeric values, precision, sensitivity and f-score values were calculated with the following equations $\underline{4.1}, \underline{4.2}$ and $\underline{4.3}$.

$$
\begin{aligned}
& \text { precision }=\frac{T P}{T P+F P} \\
& \text { recall }=\frac{T P}{T P+F N}
\end{aligned}
$$

$$
f-\text { score }=2 \times \frac{\text { precision } \times \text { recall }}{\text { precision }+ \text { recall }}
$$

Since the original boundary lines in the ground truth data are thinner and the predicted boundary lines are thicker, the pixel predictions that are not lines but within the near area of the original line are assigned as FP values and artificially increases the number of FP. In other words, since the difference in line thickness in the ground truth data with the boundary lines estimated by the models creates a much higher number of FP values compared to FN values, the recall criteria for boundary inference performance are more meaningful than the precision and f-score criteria. For this reason, especially high recall values were evaluated as the criterion for success, and it was observed that the Model IV reached the highest accuracy as explained in Table $\underline{3}$. An increase of $11 \%$ was observed in recall values, when Model I were compared to Model IV. $11 \%$ rise in recall indicates that Model IV as one of our proposed DS-HED architectures outperformed Model I representing the original HED. 
Model Train and validation loss

I

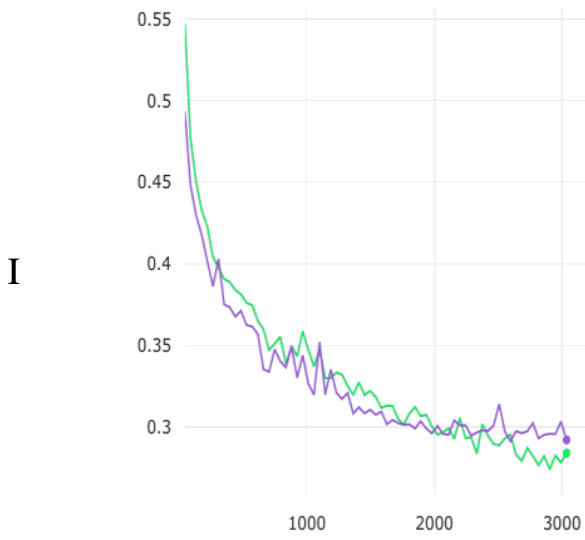

II

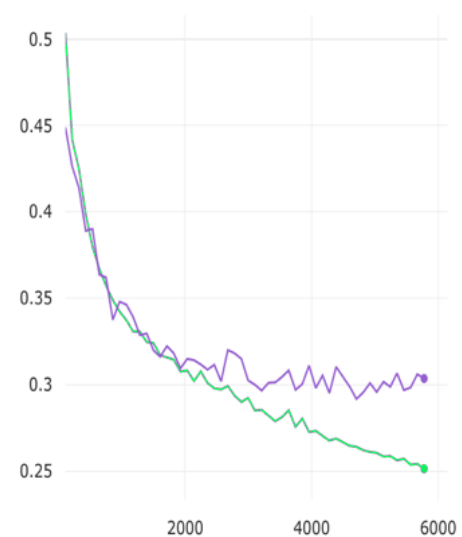

III

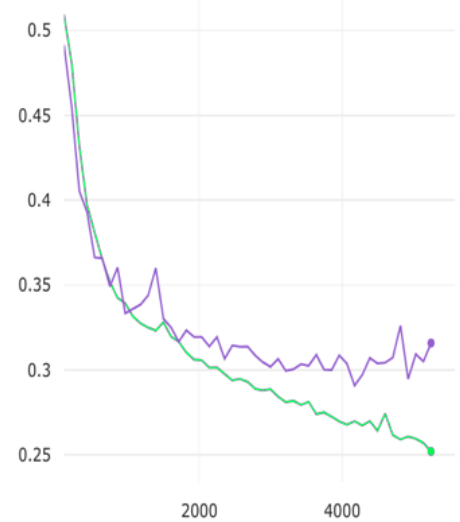

IV

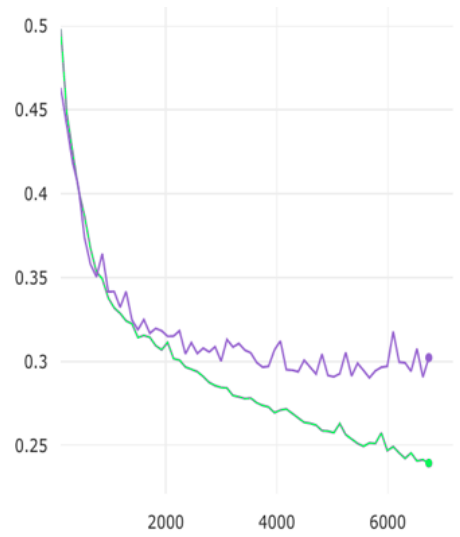

- Train loss

- Validation loss
Function error
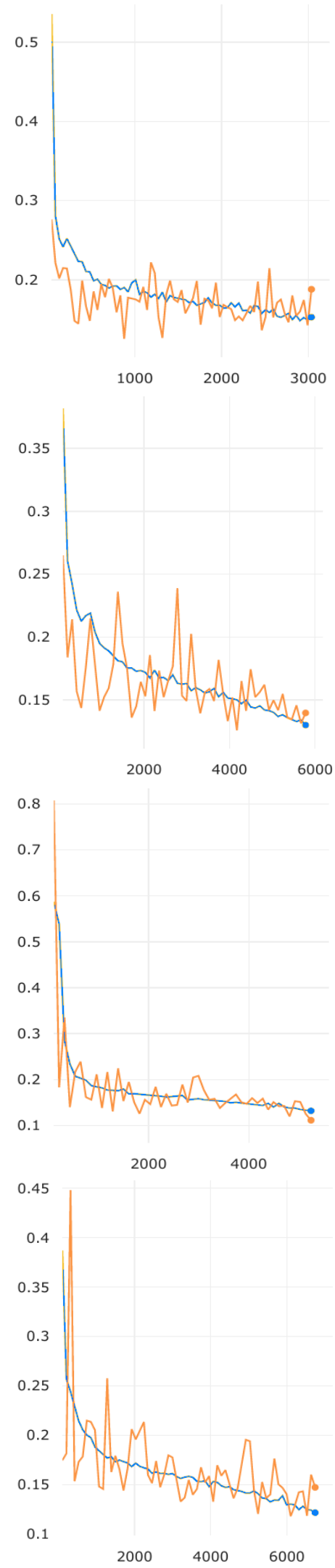

- Train function error

- Validation function error

Figure 3. The graphs of the training and validation loss for Model I, II, III and IV 
Table 3

Accuracy results for the models that were trained using layouts 2-10 and 5-12

\begin{tabular}{lllllllll}
\hline $\begin{array}{l}\text { Model } \\
\text { no }\end{array}$ & $\begin{array}{l}\text { Batch } \\
\text { size }\end{array}$ & $\begin{array}{l}\text { Training } \\
\text { data \# }\end{array}$ & $\begin{array}{l}\text { Val/Test } \\
\text { data \# }\end{array}$ & Epoch & $\begin{array}{l}\text { Time } \\
(\mathrm{min})\end{array}$ & Precision & Recall & F-score \\
\hline I & 12 & 130 & $16 / 16$ & 43 & 37 & 0.2486 & 0.7638 & 0.3751 \\
II & 6 & 130 & $16 / 16$ & 53 & 58 & 0.2391 & 0.8390 & 0.3721 \\
III & 6 & 130 & $16 / 16$ & 48 & 69 & 0.2455 & 0.8222 & 0.3781 \\
IV & 6 & 130 & $16 / 16$ & 62 & 63 & 0.1996 & 0.8779 & 0.3253 \\
\hline
\end{tabular}

The model IV, which was determined as the most successful model as a result of the comparison, was retrained by using the whole data set and the values in Figure $\underline{4}$ and Table $\underline{4}$ were obtained. Since the land use diversity in the data set is higher in the whole data set, a slight decrease was observed in the recall value compared to the previous experiment.

Model Train and validation loss

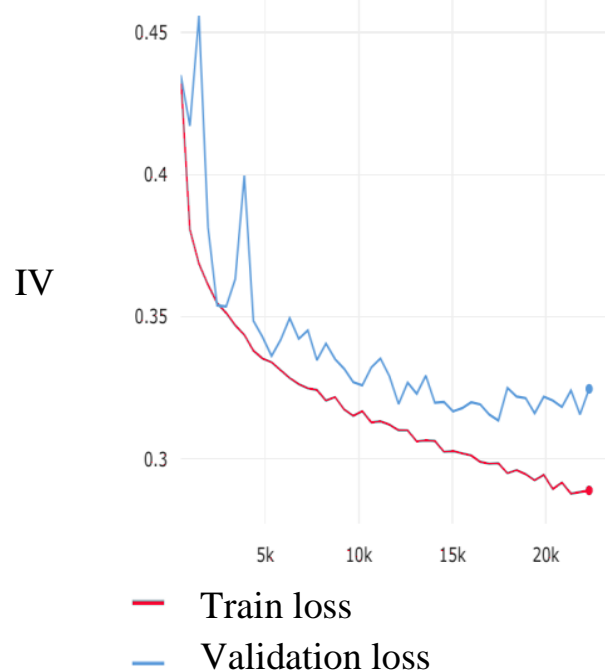

Function error

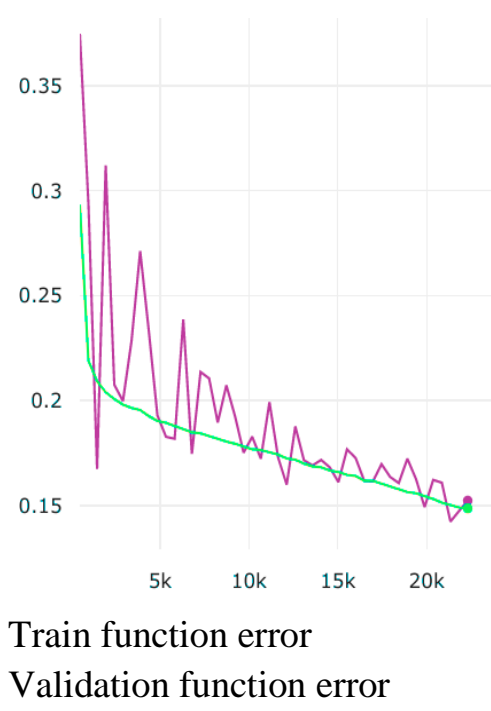

Figure 4. The graphs of the training and validation loss for Model IV based on the whole data

Table 4

Accuracy results for the models that were trained using the whole layouts

\begin{tabular}{lllllllll}
\hline $\begin{array}{l}\text { Model } \\
\text { no }\end{array}$ & $\begin{array}{l}\text { Batch } \\
\text { size }\end{array}$ & $\begin{array}{l}\text { Training } \\
\text { data \# }\end{array}$ & $\begin{array}{l}\text { Val/Test } \\
\text { data \# }\end{array}$ & Epoch & $\begin{array}{l}\text { Time } \\
(\mathrm{hr})\end{array}$ & Precision & Recall & F-score \\
\hline IV & 6 & 3120 & $384 / 384$ & 50 & 10 & 0.2724 & 0.8535 & 0.4130 \\
\hline
\end{tabular}

HED based networks produce side outputs from 800x800 pixel size patches and aggregate these side outputs to create the final boundary map. Sample five side outputs created by the retrained Model IV are shown in Figure $\underline{5}$. It is seen that different levels of detail in the image are represented in the side outputs. The final boundary map created by the combination of these five side outputs, the ground truth and the original input image is shown in Figure $\underline{6}$. 


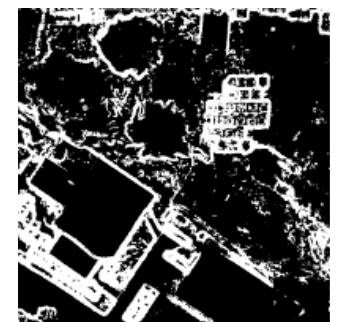

Side output I

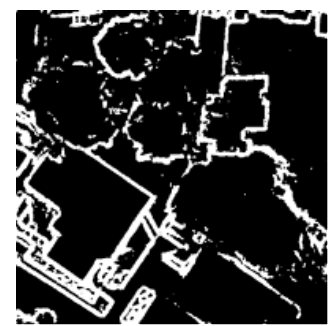

Side output II

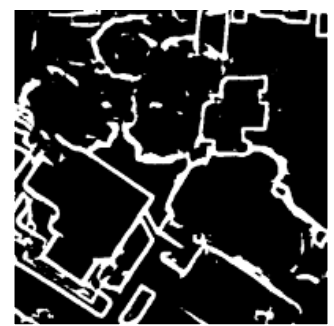

Side output III

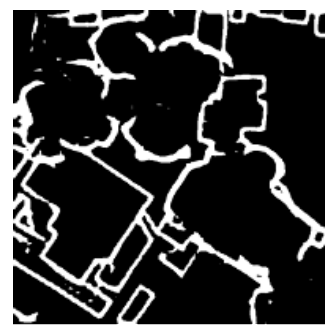

Side output IV

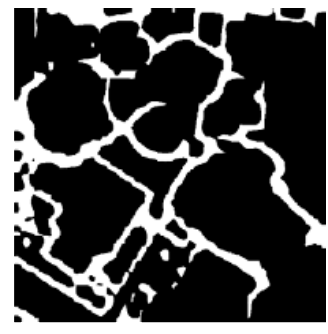

Side output $V$

Figure 5. Side outputs extracted from Model IV

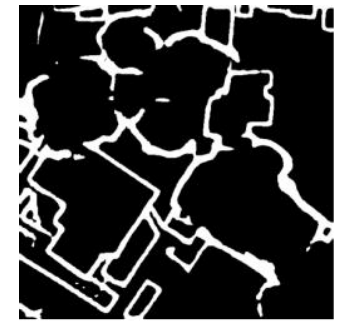

Final output

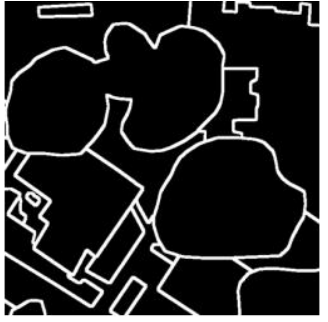

Ground truth

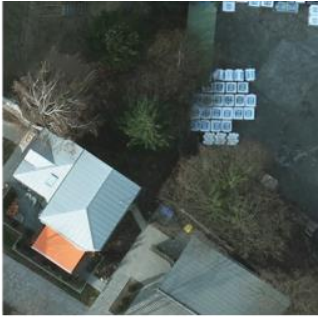

RGB image

Figure 6 . The final boundary map, ground truth and RGB image

Boundary maps were estimated from 800x800 patches from the test layouts with Model IV. All patches included in a layout are combined and a $6000 \times 6000$ boundary map is created for that layout. Figure $\underline{7}$ shows the estimated boundary map, ground truth and RGB images of the five layouts.

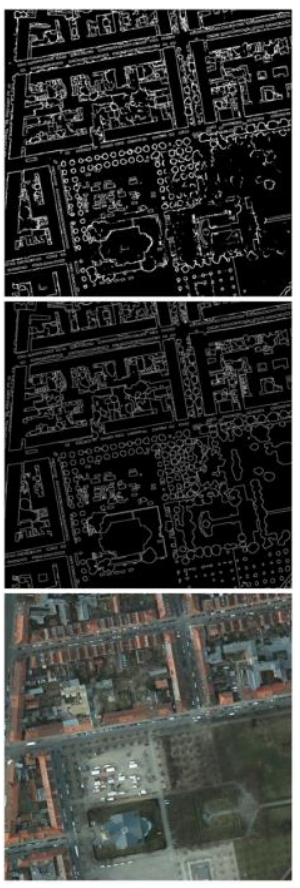

Test I
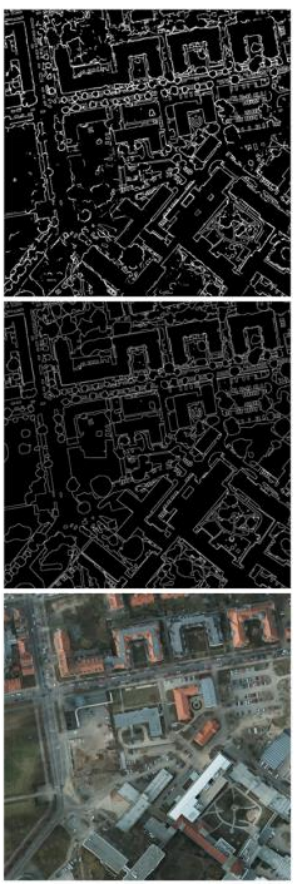

Test II
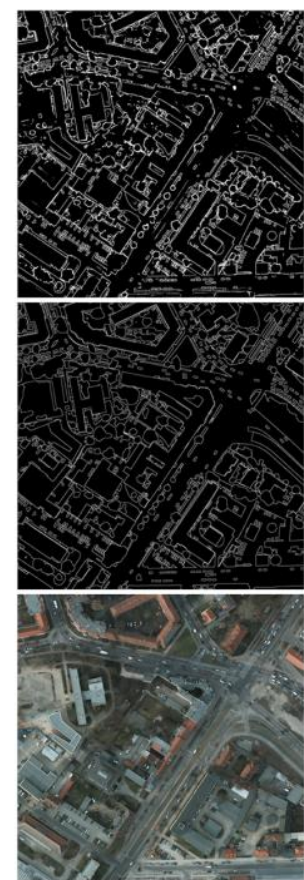

Test III
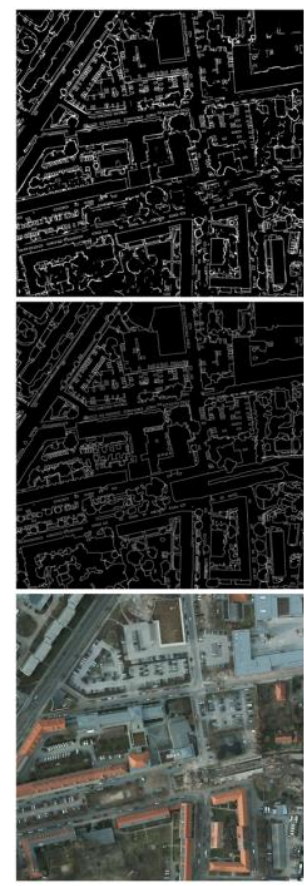

Test IV
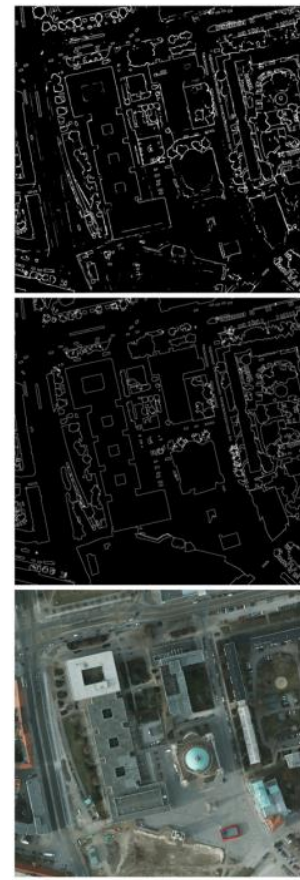

Test V

Figure 7. First row: Estimated boundaries; Second row: ground truths; Third row: RGB orthophotos. 


\section{Conclusions}

In this study, automatic border extraction, which is an important problem in remote sensing, is discussed and models have been created and trained to eliminate the deficiencies in this subject. When the results obtained from two-stream and multi-data models are evaluated, an increase in the boundary extraction performance has been achieved. This situation revealed that, except from RGB, multispectral bands, height information and vegetation information must be used as input data in boundary extraction. It has been observed that three-layer data is more advantageous than one or two layers in the second stream, since it allows transfer learning. This situation shows that training three data in each stream is an option that should be considered in future studies. On the other hand, it is important to find a balance between batch size and input size in order to use the GPU memory optimally. In the future, this problem is likely to disappear due to the improvement in graphics cards. Another problem encountered was in obtaining ground truth data. It takes a lot of time to prepare a data set that presents the boundary data accurately. For this reason, boundary extraction is prepared from semantic data with handmade methods. Both the inadequacy of the handmade methods and the border uncertainties on the images make ground truth data suspicious. Therefore, it is natural for models trained according to these ground truth data to suffer prediction losses due to data instability.

\section{Acknowledgement}

This research was supported by The Scientific and Technological Research Council of Turkey (TÜBİTAK), Project No: 119Y363.

\section{Author Contributions}

Özgün Akçay: Conceived the study, designed the paper and wrote the introduction and conclusions.

Ahmet Cumhur Kınaci: Performed data analysis and produced the statistical results and outputs.

Emin Özgür Avşar: Performed and wrote the data preparation.

Umut Aydar: Wrote the introduction.

\section{Conflicts of Interest}

The authors declare no conflict of interest.

\section{References}

Alam, M., Wang, JF., Guangpei, C., Yunrong, LV. \& Chen, Y. (2021). Convolutional Neural Network for the Semantic Segmentation of Remote Sensing Images. Mobile Networks and Applications 26(1), 200-215 Retrieved from: https://link.springer.com/article/10.1007/s11036-020-01703-3

Altınoluk E., Akçay Ö., Kınacı A. C., Avşar Ö., Polat A. B. \& Aydar U. (2020). Effects of Orthophoto Band Combinations on Semantic Segmentation. Intercontinental Geoinformation Days (IGD), (pp. 9-12). Retrieved from: http://igd.mersin.edu.tr/2020/wp-content/uploads/2020/12/final2.pdf

Bokhovkin, A. \& Burnaev, E. (2019) Boundary loss for remote sensing imagery semantic segmentation. In: Lu, H., Tang, H., Wang, Z. (eds.) ISNN 2019. LNCS, 11555, 388-401. Retrieved from: https://link.springer.com/chapter/10.1007/978-3-030-22808-8_38

Chen, L. C., Papandreou, G., Kokkinos, I., Murphy, K., \& Yuille, A. L. (2014). Semantic image segmentation with deep convolutional nets and fully connected crfs. arXiv preprint arXiv:1412.7062. Retrieved from: https://arxiv.org/pdf/1606.00915.pdf

Chen, L. C., Barron, J. T., Papandreou, G., Murphy, K., \& Yuille, A. L. (2016). Semantic image segmentation with task-specific edge detection using cnns and a discriminatively trained domain transform. Proceedings of the IEEE Conference on Computer Vision and Pattern Recognition (pp. 4545-4554). Retrieved from: https://arxiv.org/abs/1511.03328

Cheng, G., \& Han, J. (2016). A survey on object detection in optical remote sensing images. ISPRS Journal of Photogrammetry and Remote Sensing, 117, 11-28. Retrieved from: https://arxiv.org/abs/1603.06201

Dai, J., He, K., \& Sun, J. (2016). Instance-aware semantic segmentation via multi-task network cascades. 
Proceedings of the IEEE conference on computer vision and pattern recognition (pp. 3150-3158). Retrieved from: https://arxiv.org/abs/1512.04412

Guo, Y., Liu, Y., Georgiou, T., \& Lew, M. S. (2018). A review of semantic segmentation using deep neural networks. International Journal of Multimedia Iinformation Retrieval, 7(2), 87-93. Retrieved from: https://link.springer.com/content/pdf/10.1007/s13735-017-0141-z.pdf

Han, X., Zhong, Y., \& Zhang, L. (2017). An efficient and robust integrated geospatial object detection framework for high spatial resolution remote sensing imagery. Remote Sensing, 9(7), 666. Retrieved from: https://www.mdpi.com/2072-4292/9/7/666

Hariharan, B., Arbelaez, P., Bourdev, L., Maji, S., \& Malik, J. (2011). Semantic Contours from Inverse Detectors, International Conference on Computer Vision (pp. 991-998). IEEE. Retrieved from: https://ttic.uchicago.edu/ smaji/papers/contours-iccv11.pdf

Kinzie, J., \& Kuh, G. D. (2004). Going DEEP: Learning from campuses that share responsibility for student success. About Campus, 9(5), 2-8. Retrieved from: https://journals.sagepub.com/doi/abs/10.1002/abc.105

Kokkinos, I. (2015). Pushing the boundaries of boundary detection using deep learning. arXiv preprint arXiv:1511.07386. Retrieved from: https://arxiv.org/abs/1511.07386

Kokkinos, I. (2017). Ubernet: Training a universal convolutional neural network for low-, mid-, and high-level vision using diverse datasets and limited memory. Proceedings of the IEEE Conference on Computer Vision and Pattern Recognition (pp. 6129-6138). Retrieved from: https://openaccess.thecvf.com/content_cvpr_2017/papers/Kokkinos_Ubernet_Training_a_CVPR_201 7_paper.pdf

Lee, C. Y., Xie, S., Gallagher, P., Zhang, Z., \& Tu, Z. (2015, February). Deeply-supervised nets. In Artificial Intelligence and Statistics (pp. 562-570). PMLR. Retrieved from: http://proceedings.mlr.press/v38/lee15a.pdf

Maninis, K. K., Pont-Tuset, J., Arbeláez, P., \& Van Gool, L. (2017). Convolutional oriented boundaries: From image segmentation to high-level tasks. IEEE Transactions on Pattern Analysis and Machine Intelligence, 40(4), 819-833. Retrieved from: https://www.eureyecase.eu/publications/papers/2017_PAMI_ETHZ.pdf

Marmanis, D., Schindler, K., Wegner, J. D., Galliani, S., Datcu, M., \& Stilla, U. (2018). Classification with an edge: Improving semantic image segmentation with boundary detection. ISPRS Journal of Photogrammetry and Remote Sensing, 135, 158-172. Retrieved from: https://arxiv.org/abs/1612.01337

Rottensteiner, F., Sohn, G., Jung, J., Gerke, M., Baillard, C., Benitez, S., \& Breitkopf, U. (2012). The ISPRS benchmark on urban object classification and 3D building reconstruction. ISPRS Annals of the Photogrammetry, Remote Sensing and Spatial Information Sciences I-3 (2012), Nr. 1, 1(1), 293-298. Retrieved from: https://www.ipi.uni-hannover.de/fileadmin/ipi/publications/isprsannals-I-3-2932012.pdf

Shen, W., Wang, X., Wang, Y., Bai, X., \& Zhang, Z. (2015). Deepcontour: A deep convolutional feature learned by positive-sharing loss for contour detection. Proceedings of the IEEE Conference on Computer Vision and Pattern Recognition (pp. 3982-3991). Retrieved from: https://openaccess.thecvf.com/content cvpr 2015/html/Shen_DeepContour_A Deep 2015 CVPR pa per.html

Shorten, C., \& Khoshgoftaar, T. M. (2019). A survey on image data augmentation for deep learning. Journal of Big Data, 6(1), 1-48. Retrieved from : https://journalofbigdata.springeropen.com/articles/10.1186/s40537-019-0197-0

Xie, S., \& Tu, Z. (2015). Holistically-nested edge detection. Proceedings of the IEEE International Conference on Computer Vision (pp. 1395-1403). Retrieved from: https://ieeexplore.ieee.org/document/7410521

Yu, Z., Feng, C., Liu, M. Y., \& Ramalingam, S. (2017). Casenet: Deep category-aware semantic edge detection. Proceedings of the IEEE Conference on Computer Vision and Pattern Recognition (pp. 59645973). Retrieved from: https://arxiv.org/abs/1705.09759

Yuan, X., Shi, J. \& Gu, L. (2021). A review of deep learning methods for semantic segmentation of remote sensing imagery, Expert Systems with Applications, 169, 114417. Retrieved from: https://www.sciencedirect.com/science/article/abs/pii/S0957417420310836 